\title{
Hepatitis B virus genomic nucleic acid in the activation and maturation of bone marrow-derived dendritic cells
}

\author{
Chean Ring Leong ${ }^{a^{*}}$, Tsukasa Seya ${ }^{b}$, Woei Yenn Tong ${ }^{a}$, Wen-Nee Tan ${ }^{c}$ \\ ${ }^{a}$ Department of Bioengineering, Universiti Kuala Lumpur, Malaysian Institute of Chemical and Bio-engineering Technology, Lot 1988, Kawasan \\ Perindustrian Taboh Naning, 78000 Alor Gajah, Melaka, Malaysia \\ ${ }^{b}$ Department of V accine Immunology, Hokkaido University Graduate School of Medicine, Sapporo, Japan \\ 'School of Distance Education, Universiti Sains Malaysia, Minden, Penang, Malaysia
}

Received 19th August 2021 / Accepted 25th November 2021

\begin{abstract}
Hepatitis B virus (HBV) is the etiological agent that causes a self-limiting or chronic infection in the hepatocytes of about 250 million people worldwide. The role of adaptive immune system during HBV infection has been well studied. However, the innate immune system's responses against HBV during the early stage of infection largely remain unclear. In this study, we found that HBV genomic DNA or Salmon Sperm DNA (SSD) was able to induce the innate immune response in the macrophages cell line RAW264.7 but not the hepatocyte cell line, HepG2, indicating that hepatocytes may lack of a functional DNA-sensing pathway and hence are unable to respond to the presence of foreign DNA in the cytosol with type 1 IFN response. Thus, we hypothesized that non-parenchymal cells like the Antigen Presenting Cells (APC) might be crucial in triggering the initial immune response to suppress the virus replication and link the innate and adaptive responses. Using bone marrow-derived DCs (BMDC) as a model, this study demonstrated that HBV genomic DNA is able to induce cytokines like TNF-alpha, IL-6, and IL$12 \mathrm{p} 40$ secretion. We also examined the activation and maturation of BMDCs when exposed to the HBV genomic DNA intracellularly and extracellularly. A significant shift of $\mathrm{CD} 86^{+}$and $\mathrm{CD} 40^{+}$cell populations was observed during extracellular exposure of BMDC to Poly I:C and HBV genomic DNA, indicating that TLRs may be vital in the uptake of the extracellular viral DNA to activate the BMDCs. Moreover, transfection of intracellular nucleic acid stimuli, including HBV genomic DNA as well induced BMDCs maturation. Our findings highlight the critical function of DCs in antiviral response as a potential connection between the innate and adaptive immune systems during HBV pathogenesis. Nevertheless, further study is required to determine the role of cytosol DNA sensing pathway in DCs during HBV infection.
\end{abstract}

Keywords: ANIb, GGDC, MLSA, Next-generation Sequencing, Pseudomonas nitroreducens

\section{INTRODUCTION}

Chronic Hepatitis B is HBV infection that can cause cirrhosis, liver failure, and hepatocellular carcinoma (HCC) (Rehermann \& Nascimbeni, 2005). Chronic hepatitis B affects over 30 million individuals worldwide, making it a major global health problem. Hepatitis B prevalence in SouthEast Asia is $2 \%$, according to the World Health
Organization (WHO 2017). Meanwhile, over 1.1 million people in Malaysia are chronically infected with hepatitis B, with a $4.7 \%$ prevalence of HBsAg (Yin and Tong, 2006).

HBV is a member of the Hepadnaviridae family with $3.2 \mathrm{~kb}$ of partly circular and relaxed doublestranded DNA, and it only replicates in the

*Author for correspondence: Chean Ring Leong, Department of Bioengineering, Universiti Kuala Lumpur, Malaysian Institute of Chemical and Bio-engineering Technology, Lot 1988, Kawasan Perindustrian Taboh Naning, 78000 Alor Gajah, Melaka, Malaysia.

Email_crleong@unikl.edu.my 
hepatocytes of primates (Ganem \& Prince, 2004). Even though only approximately $5 \%$ of $\mathrm{HBV}$ infected patients develop chronic infections that lead to liver cirrhosis and hepatocellular carcinoma (HCC), HBV is responsible for roughly $20 \%$ of HCC deaths (Zuckerman \& Zuckerman, 2000).

During HBV infection, the role of adaptive immune responses has been clearly demonstrated with the presence of $\mathrm{HBV}$-specific $\mathrm{CD}^{+}$and $\mathrm{CD}^{+} \mathrm{T}$ cells, a vital component of the immune response against HBV (Phillips et al., 2010). Patients with acute HBV infection have been reported with efficient development of B-cell antibody production against HBV (Rehermann \& Nascimbeni, 2005). However, the significance of innate immunity during $\mathrm{HBV}$ infection is still debatable since it is impossible to diagnose individuals at an early stage of infection. Furthermore, there is no reliable cell-based in vitro infection method or practical animal model.

During the early stage of infection, the host's innate immune system is the first line of defence against $\mathrm{HBV}$. HBV is thought to be a stealth virus that only activates the innate immune response to a limited extent as reported previously that HBV did not induce any cellular gene expression in the hepatocytes. (Wieland \& Chisari 2005). There is more accumulating evidence that $\mathrm{HBV}$ can activate innate immune responses. Recently, Zhang et al. (2020) showed that primary human hepatocytes are able to sense $\mathrm{HBV}$ particles via TLR2 leading to an activation of anti-HBV immune responses in vitro. These findings challenge the previously described stealth properties of HBV. Furthermore, the importance of circulating natural killer (NK) cells in the liver against viral hepatitis is becoming more evident (Zhang et al., 2016). Viral infection generally activates two different signalling pathways that result in the production of type I interferons (e.g., IFN-alpha and IFN-beta) and other proinflammatory cytokines (Leong et al., 2015). Our previous study also showed that interferoninduced genes like the ISG20 degrade the RNA of the virus to impede HBV replication in vitro and in vivo (Leong et al., 2016). These studies suggest that the innate immune response is crucial in suppressing HBV replication at the initial stage of infection, particularly in the self-limiting HBV infection. However, it remains unclear how the viral nucleic acid was recognized to initiate the innate immune responses. A subfamily of Tolllike receptors (TLR3, 7, 8, and 9) and the retinoic acid-inducible gene I (RIG-I) have been suggested to detect HBV double-stranded RNA (dsRNA) in the cytosol (Megahed et al., 2020). However, using a range of knockout mice deficient in specific pathways of the innate system, we have previously shown that RNA-sensing pathways do not participate in the regulation of HBV replication in a mouse model; meanwhile, MyD88 is implicated in the HBV clearance (Leong et al., 2015). Since the cytosolic DNA sensing pathway was only first discovered recently, there are limited studies on the recognition of HBV DNA, particularly in the antigen-presenting cells (APCs) (Kato et al., 2011; Keating et al., 2011)

Dendritic cells (DCs) and macrophages are among the APCs that process antigen material to activate the immune system's natural killer (NK) or $\mathrm{T}$ cells and $\mathrm{B}$ cells (Banchereau \& Steinman, 1998). During viral or bacterial infection, identifying pathogen-associated molecular (PAMPs) by immature DCs causes their activation and subsequent maturation (Buchta \& Bishop, 2014; Kumar et al., 2009). DCs are then responsible for producing cytokines that influence T-cell polarization and NK cells maturation; thus, they are also known as the messengers between the innate and adaptive immune systems. The role of DCs in triggering the innate immune response during the early stage of HBV infection remains unclear.

During the life cycle of HBV, the host machinery will transcribe four open reading frames (ORFs): HBV polymerase, the HBV core protein $(\mathrm{HBc} / \mathrm{HBe} \mathrm{Ag})$, the surface proteins ( $\mathrm{HBs}$ $\mathrm{Ag}$ ), and the regulatory protein ( $\mathrm{HBx} \mathrm{Ag}$ ) (Seeger \& Mason, 2000). The pregenomic RNA (pgRNA) will be transformed into partially double-stranded relaxed circular DNA ( $\mathrm{rCDNA}$ ), which will be used to make the virus particles' genetic material. Unlike most viruses that employ DNA or RNA as their genetic material, $\mathrm{HBV}$ has a unique life cycle. The viral RNA must undergo reverse transcription to generate double-stranded rcDNA before being packaged into an infectious virus particle. Thus, the viral nucleic acid can be found in single-stranded RNAs, double-stranded RNAs, and double-stranded DNA during the life cycles of HBV in the host cell. The phase of these viral 
nucleic acids that stimulates the host's innate immune responses is unknown.

We hypothesized that the absence of innate response in hepatocyte is partly due to the lack of DNA sensing pathway. Thomsen et al. 2016 reported that both murine and human hepatocytes lack of DNA of the cellular sensing pathway, cGAS-STING pathway. Hence, in the present study, we investigated the role of $\mathrm{HBV}$ genomic DNA in the maturation and activation of the bone marrow-derived DCs' immune response. Maturation and activation of DCs triggered by the HBV genomic DNA will provide crucial information in understanding the viral pathogenesis.

\section{MATERIALS \& METHODS}

\section{Animals}

Female C57BL/6J mice were purchased from SANKYO LABO SERVICE and used at 7-9 weeks of age with 3 mice in each group $(n=3)$. All mice were maintained under specific pathogenfree conditions in the Animal Facility at Hokkaido University Graduate School of Medicine (Sapporo, Japan). All experiments were approved by the Institutional Animal Care Committee. The study was approved by the local ethics committee and conducted according to the guidelines for the Care and Use of Laboratory Animal of Hokkaido University (approved number: 17-0032).

\section{Cell culture and transfection with nucleic acid stimuli}

RAW264.7 and HepG2 cells were obtained from the ATCC and maintained in RPMI1640 and Dulbecco's modified Eagle medium (DMEM) respectively supplemented with 10\% FBS and antibiotics (Seeger \& Mason, 2000). The RAW264.7 and HepG2 were seeded on the 24well and cultured for $24 \mathrm{~h}$ before transfected with Poly I:C, Salmon Sperm DNA (SSD), control vector (pTRE2) and the plasmid containing the HBV full genome (pTRE2-HBV) using Lipofectamine 2000 (Life technologies) according to the manufacturer's instruction. After $12 \mathrm{~h}$ of incubation, the total RNA of the cells was isolated with Trizol and subjected to qPCR analysis later on.

\section{Reagents}

Polyinosinic: polycytidylic acid (poly I:C) and salmon sperm DNA (SSD) were purchased from Amersham and Sigma-Aldrich, respectively. The plasmid carrying the $1.4 \times \mathrm{HBV}$ genome (pTER2HBV), in which HBV RNA was transcribed from the pCMV minimal promoter, was kindly gifted by Kazuaki Chayama. The construction of wildtype HBV 1.4 genome length, pTRE-HBwt, had been described previously (GenBank accession no. AB206816) (Tsuge et al., 2005). HBV genomic DNA used in the study was the amplified PCR fragments of HBV's full-length genomic DNA using pTRE2-HBV as a template.

\section{Generation of bone marrow DCs}

Bone marrow cells were used to generate the bone marrow DCs (BMDCs) with the method previously reported (Inaba et al., 2009). Briefly, the bone marrow cells were isolated from the femur and tibia of the mice aseptically. $2 \times 10^{6}$ cells were seeded into each well and cultured in RPMI 1640 medium containing 10\% fetal calf serum (FCS). To stimulate the maturation and proliferation of the bone marrow cells, $10 \mathrm{ng} / \mathrm{mL}$ of recombinant murine GM-CSF (R\&D Systems) was added to the culture medium. To test the effect of the HBV genomic DNA, the BMDC were either incubated with the viral genomic DNA or transfected with the viral genomic using Lipofectamine2000 and electroporation. After being stimulated with the viral genomic DNA for $24 \mathrm{~h}$, the BMDC was harvested for FACS to analyze the CD-86 and CD-40 surface cell markers or cytokines analysis.

\section{Quantitative RT-PCR (qPCR)}

Dnase-I-treated total cellular RNA was used to generate cDNA by SuperScript III Reverse Transcriptase (Life Technologies). Real-time PCR was performed with SYBR Green Master (Roche) and the LightCycler 480 System (Roche) by using the following IFN-beta and HBV-specific primers (Table 1). The gene expression data were normalized with reference to the level of GAPDH in the same samples. 
Table 1. Primers used for qPCR.

\begin{tabular}{|c|c|}
\hline \multirow{2}{*}{ IFN- $\beta$} & $\begin{array}{l}\text { F: 5' -CCA GCT CCA AGA AAG } \\
\text { GAC GA - } 3^{\prime}\end{array}$ \\
\hline & $\begin{array}{l}\text { R: 5'- CGC CCT GTA GGT GAG } \\
\text { GT'T AT-3' }\end{array}$ \\
\hline \multirow{2}{*}{$\mathrm{HBV}$} & $\begin{array}{l}\text { F: 5'- TTC CTC TTC ATC CTG } \\
\text { CTG CT -3' }\end{array}$ \\
\hline & $\begin{array}{l}\text { R: GTC CCG TGC TGG TAG TTG } \\
\text { AT }-3^{\prime}\end{array}$ \\
\hline
\end{tabular}

\section{Cytokines analysis}

After exposing the BMDC to the HBV genomic DNA and other nucleic acid stimuli, the culture medium was collected and analyzed for cytokine production. TNF-alpha, IL-6, and IL-12p40 were analyzed with BD CBA Flex set.

\section{Cell population and co-stimulation molecule analysis}

After stimulation with HBV genomic DNA, the surface cell marker of the BMDC was analyzed in a FACScan flow cytometer (Becton Dickinson, Mountain View, CA, USA). Briefly, the BMDC after $24 \mathrm{~h}$ of exposure to the HBV genomic DNA was incubated with specific labeled monoclonal antibodies, anti-CD86 and anti-CD40. Cytometry data were analyzed using WinMDI $2 \cdot 8$ software.

\section{Statistical analysis}

The statistical significance of the obtained data in this study was analyzed using a two tail unpaired $t$ test and $\mathrm{p}<0.05$ was regarded as statistically significant.

\section{RESULTS AND DISCUSSION}

\section{Transfection of $\mathrm{HBV}$ genomic $\mathrm{DNA}$ and other DNA stimuli upregulate IFN-beta expression in macrophages cell line (RAW264.7) but not hepatocytes (HepG2)}

To investigate the role of innate DNA sensing during HBV infection, we transfected the human hepatocyte cell line HepG2 with the plasmid containing HBV full genomic DNA and other nucleic acid stimuli, including Poly I:C SSD and control vector. The IFN-beta expression of the host cells is often a signature response of the innate immune system as the first line of defense against viruses. As shown in Figure 1(A) that only
Poly I:C, a double-stranded RNA analog, triggered an up-regulation of IFN-beta expression in the hepatocytes while other DNA stimuli, including SSD and a plasmid containing HBV full genomic DNA, failed to induce the expression of IFN-beta gene. The results obtained are in good agreement with Thomsen et al. (2016) that both murine and human hepatocytes lack of DNA of the cellular sensing pathway, cGAS-STING pathway. Because hepatocytes lack STING expression, they lack a functional DNA-sensing pathway and are hence unable to respond to the presence of foreign DNA in the cytosol with a type 1 IFN response. Hepatocytes' ability to manage HBV infection appears to be hampered by a lack of DNA sensing. As a result, we believe that hepatocytes are an advantageous biological habitat for DNA viruses. This is especially beneficial for a tiny DNA virus like HBV, which is expected to have fewer immune-evasion mechanisms than larger viruses like herpesviruses and poxviruses (Bowie, 2008). Next, we transfected RAW264.7 cells, a macrophages cell line with identical nucleic acid stimuli, and determined the IFN-beta expression. As shown in Figure 1(B), contrary to the HepG2 cellular response, the IFN-beta expression is upregulated with the presence of all the stimuli in the cytoplasm of RAW264.7 cells. These two cell lines exhibit distinct responses when transfected with the stimuli. Thus, such results indicated that HBV might only be a 'stealth' virus in the infected host cell of hepatocytes due to a lack of a pathway to sense the presence of HBV DNA. We also examined the $\mathrm{HBc}$-antigen expression in both HepG2 and RAW264.7 cells and found that the expression of $\mathrm{HBc}$ in HepG2 is much prominent compared to RAW264.7 cells (Supplementary Data). The result also indicates that hepatocytes are an advantageous host cell for HBV to support its replication.

Other infiltrating and circulating immune cells, such as antigen-presenting cells (APCs), including DCs and macrophages, may respond to the presence of HBV viral DNA upon infection at the early stage and secrete the crucial cytokine to suppress the replication of HBV in the infected host cells. Our previous study also reported that $\mathrm{HBV}$-infected hepatocytes release exosomes containing HBV nucleic acids to the nonparenchymal cells like macrophages and DCs in 
vivo during the innate immune response to $\mathrm{HBV}$ (Kouwaki et al., 2016)

\section{$H B V$ genomic DNA induced various cytokines production in BMDCs}

To investigate the immune responses of APCs during exposure to $\mathrm{HBV}$ genomic DNA, the generated BMDCs was transfected with Poly I:C, SSD (SSD), control plasmid (pTRE), the plasmid containing the complete genomic sequence of HBV (pTRE-HBV) as well as the full genomic DNA of HBV. After $24 \mathrm{~h}$, the cytokines such as TNF- $\alpha$, IL- 6 , and IL-12p40 secreted by the BMDC were analyzed. As shown in Figure 2(AC), HBV genomic DNA triggers a significant amount of all the cytokines secreted into the culture medium compared to the other stimuli. $\mathrm{TNF}-\alpha$ is a prominent antiviral cytokine that stimulates adaptive immunity and the antiviral effects of CTLs (Phillips et al., 2010). The result obtained agrees with the findings reported by Tzeng et al., 2014 that TNF receptor blocker delayed the HBV viral clearance and increased the expression of inhibitory receptor, programmed death-1 (PD-1) in CD8 ${ }^{+} \mathrm{T}$ cells. Also, Hösel et al. (2009) reported that IL-6 plays a vital role in the early phase of infection to control the virus's spread. IL-6 has been shown to prevent the death of $\mathrm{HBV}$-infected hepatocytes and limit the activation of adaptive immune responses during the early phase of infection. Whereas IL-12, as an immunomodulatory cytokine, plays an essential role in promoting cellular immunity. Bertoletti and Ferrari (2013) suggested that IL-12 is crucial in inducing $\mathrm{B}$ cell responses resulting in IFN- $\gamma$ and anti-HBs antibody production. The results indicated that non-parenchymal cells in the liver, such as DCs, can secret various antiviral cytokines when exposed to the HBV genomic DNA. This is consistent with the finding of Kouwaki et al. (2016) whereby they reported that hepatic NK cells were activated during the HBV infection in the tree shrew animal model. DCs as the antigenpresenting cell may act as messengers between the innate and adaptive immune systems. Indeed, DC-mediated NK cell activation aids in the development of effective innate immunity (Oth $e t$ al., 2016).

\section{(A)}

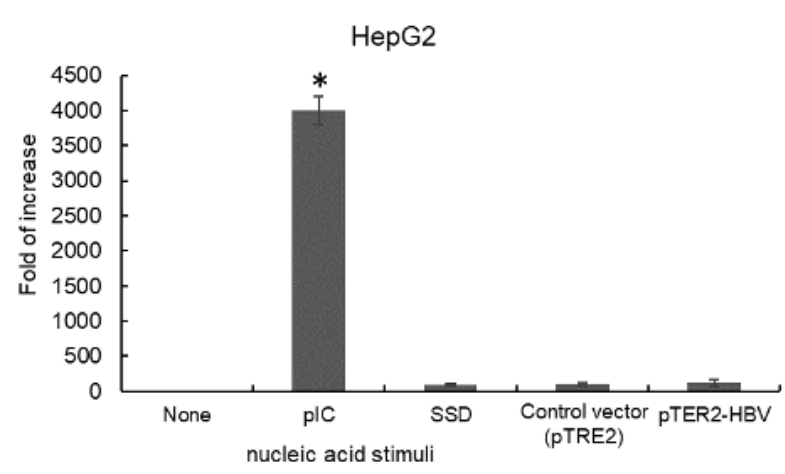

(B)

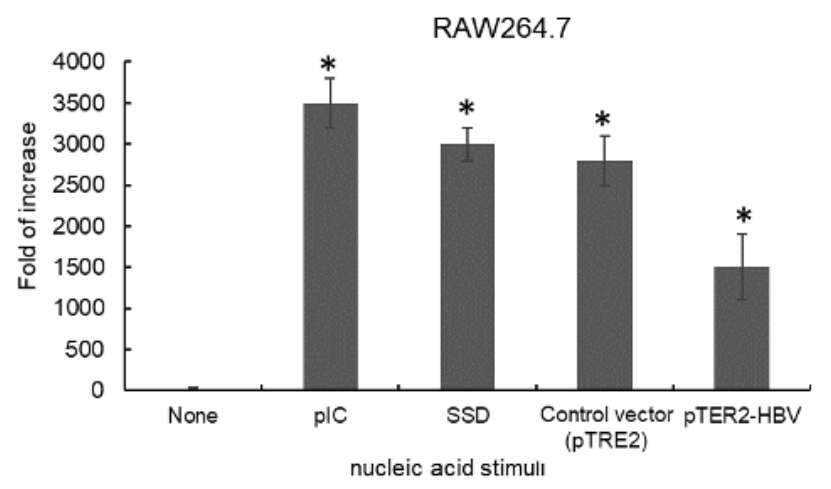

Figure 1. The IFN-beta mRNA induction in hepatocytes and macrophages cell lines in response to HBV genomic DNA and other nucleic acid stimuli. (A) HepG2 cells were transfected with Poly I:C (pIC), SSD (Salmon Sperm DNA), control vector (pTRE2), and pTER2-HBV, RT-PCR analysis was performed to determine the expression levels of IFN-beta. (B) RAW264.7 cells were transfected with Poly I:C (pIC), SSD (Salmon Sperm DNA), control vector (pTRE2), and pTER2-HBV, RT-PCR analysis was performed to determine the expression levels of IFN-beta. The IFN-beta expression was normalized to GAPDH expression. Data are presented as mean $\pm S D(n=3)$. (Asterisks indicate a statistically significant difference between the group in comparison to the none nucleic acid stimuli group as determined by Student's t-test $* p$-value $<0.005)$. 
(A)

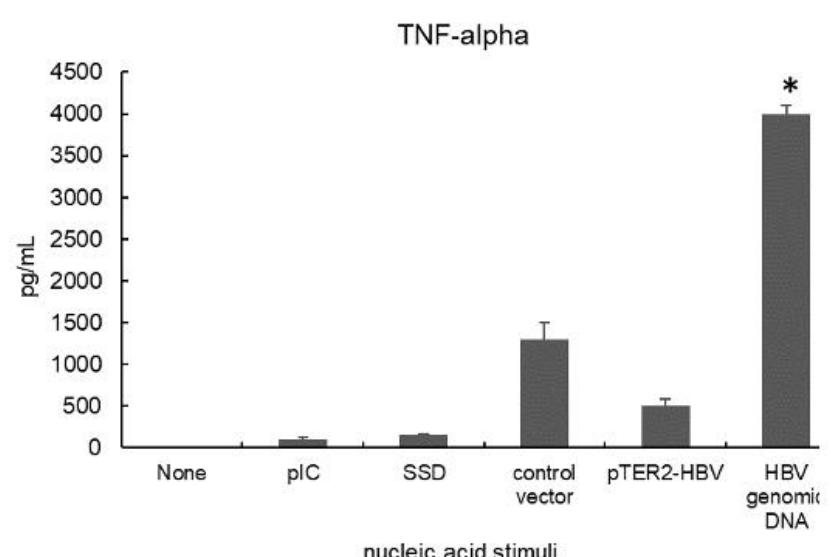

(C)

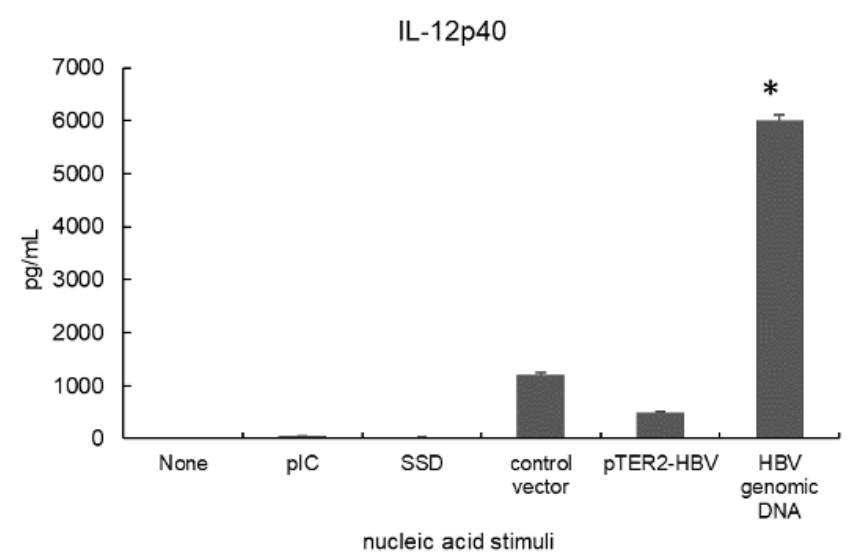

(B)

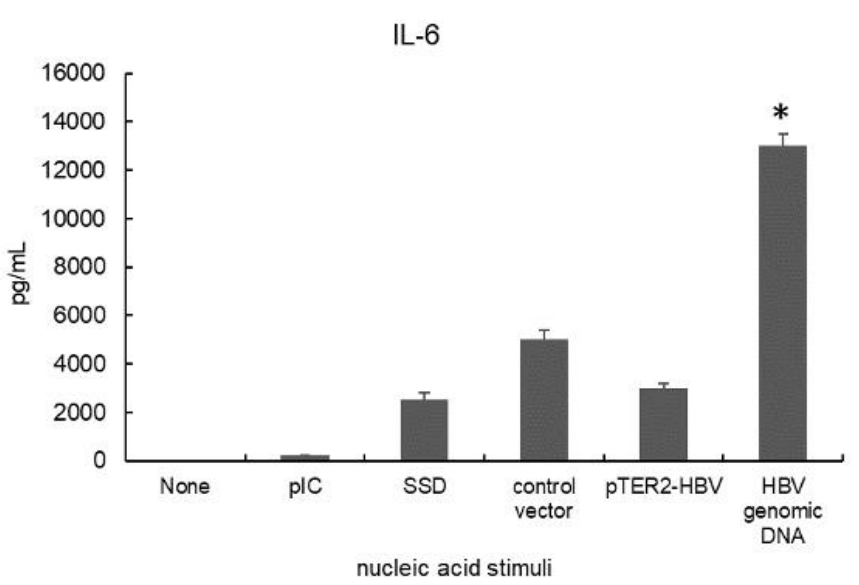

Figure 2. The murine bone marrow-derived DCs were seeded onto a 24-well plate and cultured for $24 \mathrm{~h}$. The various nucleic acid stimuli were added to the BMDC, and the cytokines in the culture medium were analyzed. (A) TNF-alpha, (B) IL-6, and (C) IL-12p40 secreted by the Bone marrow-derived DCs after transfected with the stimuli was measured. Data are presented as mean \pm SD $(n=3)$. (Asterisks indicate a statistically significant difference between the group in comparison to the none nucleic acid stimuli group as determined by Student's t-test $* p$-value $<0.005)$.

\section{Maturation of BMDC during exposure to extracellular $\mathrm{HBV}$ genomic $\mathrm{DNA}$}

After maturation, immature DCs with an extended and adherent phenotype and podosomes shift to a rounded and non-adherent migratory phenotype (Burns et al., 2004). Increased surface expression of MHC Class II (MHCII) and co-stimulatory molecules such as CD80, CD86, and CD40, among others, accompany these morphological alterations (Reise, 2006). To study the effect of extracellular HBV genomic DNA on the maturation of BMDC, the BMDCs were cultured with various nucleic acid stimuli, including pIC, SSD, pTRE2-
$\mathrm{HBV}$, and the HBV genomic DNA. Upon $24 \mathrm{~h}$ incubation, the BMDCs were stained with antibodies specific to the cell surface marker of CD86 and CD40. As shown in Figure 3 (A) and (B), the extracellular exposure of the BMDCs to $\mathrm{pIC}$ and $\mathrm{HBV}$ genomic DNA causes a significant increase of the $\mathrm{CD}_{86}{ }^{+}$and $\mathrm{CD} 40^{+} \mathrm{BMDC}$ population $(95.2 \%$ and $97.2 \%$ for PolyI:C while $78.6 \%$ and $58.6 \%$ for HBV genomic DNA, respectively). However, the significant shift in the BMDC population is not observed when incubated with SSD (SSD) or pTRE2-HBV. The result obtained is similar to previous findings by Barton et al. (2006), whereby the DNA of salmon 
sperm did not activate the Toll-Like Receptors (TLRs) and thus serve as a negative control for the TLRs induction experiments. The results obtained indicate that the presence of $\mathrm{HBV}$ genomic DNA could trigger the maturation and activation of BMDC, possibly via the engagement with TLRs in a similar manner with PolyI:C when present in the extracellular environment. The PolyI:C and HBV genomic HBV uptake into the BMDC might occur via the endosome's or phagosome action, thus triggering the activation on set for the TLRs such as TLR-9 or TLR-3. In the context of sensing extracellular nucleic acid, available evidence indicates that TLR3, TLR7, and TLR9 that can recognize viral and bacterial nucleic acids in the APCs like DCs are localized in intracellular compartments (Funami et al., 2004; Latz et al., 2004). Matsumoto et al. (2014) have reported that TLR3 is a type I transmembrane protein found in myeloid DCs (DCs) and fibroblasts/epithelial cells located in the endosomal membrane. Extracellular viral doublestranded RNA (dsRNA) and synthetic dsRNA, poly(A)-dsRNA, is recognized by TLR3.

On the other hand, TLR 9 expressed in endosomal membranes was identified as the first DNA receptor recognizing hypomethylated CpG motifs (Wagner et al., 2000). TLR9 expression is limited to B cells and DCs in humans (Kadowaki et al., 2001). Thus, DNA-induced immune responses in other cell types might not be activated via TLR9. The results obtained are in good agreement with our previous finding that extracellular vehicles (EVs) released from HBVinfected hepatocytes enclosed viral nucleic acids and triggered immune responses in macrophages (Kouwaki et al., 2016). DCs may as well be a trigger in a similar manner as the macrophages.

TLRs are essential in helping the host sense invading pathogens like microorganisms by recognizing the highly conserved structural motifs known as pathogen-associated microbial patterns (PAMPs). Bacterial DNA and viral doublestranded RNA are the common PAMPs that trigger the host's innate immune responses. The stimulation of TLRs by PAMPs will initiate the signaling cascade like MyD88 and IRAK, leading to the activation of transcription factors (Barton et al., 2006). The secretion of pro-inflammatory cytokines and effector cytokines will be the result that activates the adaptive immune response.

\section{Maturation of BMDC during exposure to intracellular $\mathrm{HBV}$ genomic DNA}

We also investigated the maturation of BMDC when exposed to the intracellular nucleic acid stimuli by transfecting the nucleic acid stimuli, including PolyI:C, SSD, plasmid containing HBV, and HBV genomic DNA, into the cells. As shown in Figure 4 (A) and (B), a significant shift of the $\mathrm{CD}^{\circ} 6^{+}$and $\mathrm{CD} 40^{+}$expressing BMDC population was detected when the cells were exposed to all the intracellular nucleic acid stimuli. The results obtained indicated that the presence of the nucleic acid including HBV genomic DNA in the cytoplasm of the DCs, will trigger its activation and maturation. This finding is parallel with numerous reports that DNA is generally found in the nucleus of eukaryotic cells, and its presence in other places, such as the cytoplasm and endosomes, is thought to activate the immune system. (Lund et al., 2003; Ishii et al., 2006; Stetson \& Medzhitov, 2006; Kerur et al., 2011). Nevertheless, the result obtained in this study is still insufficient to suggest that the cytosolic DNA sensing pathway is $\mathrm{HBV}$-specific to trigger the immune responses of DCs.

Unlike TLRs that have been well identified and characterized as pattern recognition receptors (PRRs) that respond to nucleic acid in the endosomes or extracellular, cytosolic PRRs which sense viral and host nucleic acids in the cytoplasm have only recently been discovered (Kato et al., 2011; Keating et al., 2011). Among recent works on the role of cytosol DNA sensing pathway during viral infection, Horan et al. (2013) reported that DNA viruses like the herpes simplex virus (HSV) capsid were ubiquitinated in the cytoplasm and destroyed by the proteasome, resulting in DNA release into the cytoplasm. Such mechanisms were reported in macrophages, one of the APCs (Horan et al., 2013). Thus, further research is required to elucidate the cytoplasmic DNA sensing mechanisms in the context of HBV. 
(A)

None
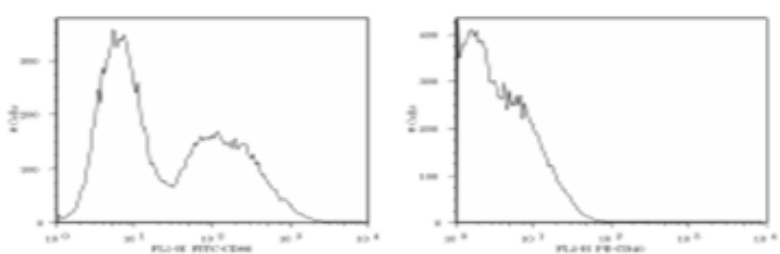

plC
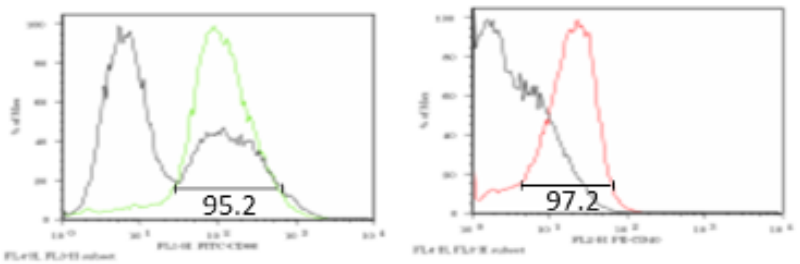

Salmon sperm

DNA
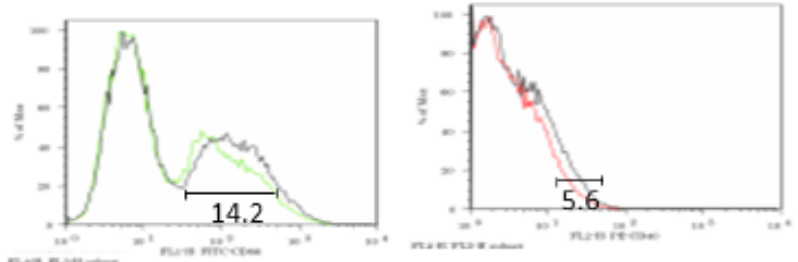

pTRE2-HBV
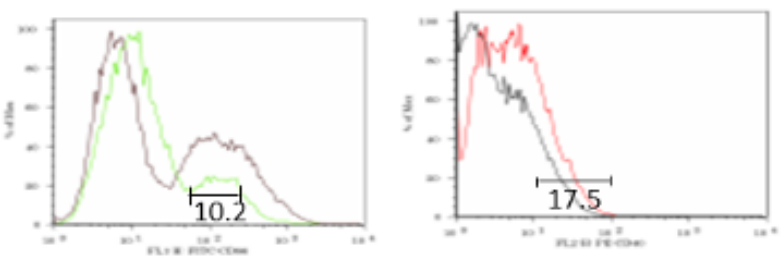

HBV genomic

DNA
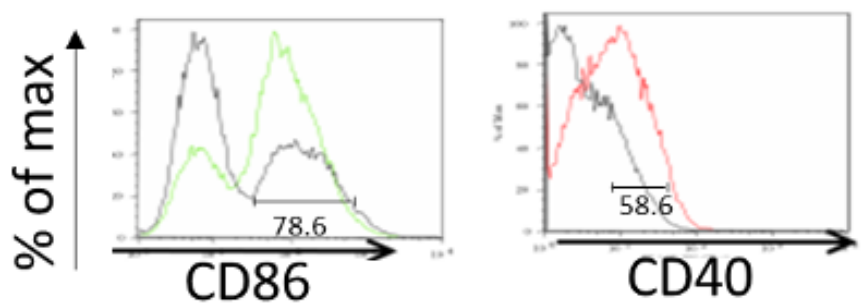

(B)

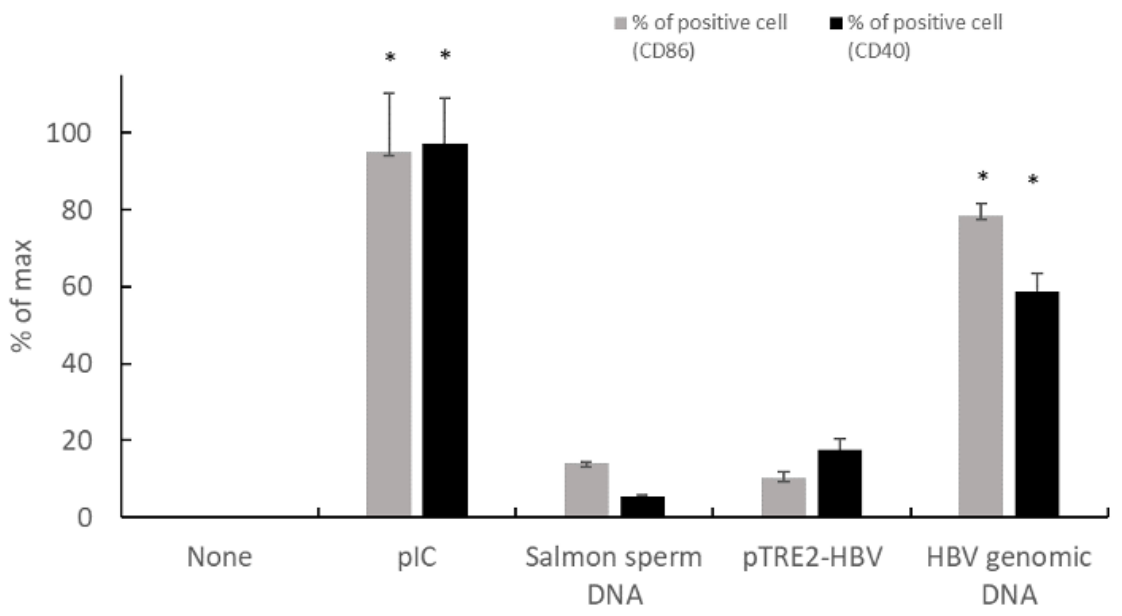

Figure 3. Exposure of BMDCs to extracellular HBV genomic DNA and a plasmid containing the HBV full genome moderately mediate the maturation of the DCs. (A) Representative flow cytometry histograms show the $\mathrm{CD}^{+} 6^{+}$(green) and $\mathrm{CD}^{4} 0^{+}$(red) BMDC population after exposure to the extracellular environment's nucleic acid stimuli. (B) Summarized significance was determined (Asterisks indicate a statistically significant difference between the group in comparison to the none nucleic acid stimuli group as determined by Student's t-test $* p$-value $<0.05)$. 
(A)

\section{(A)}

$\mathrm{plC}$

None
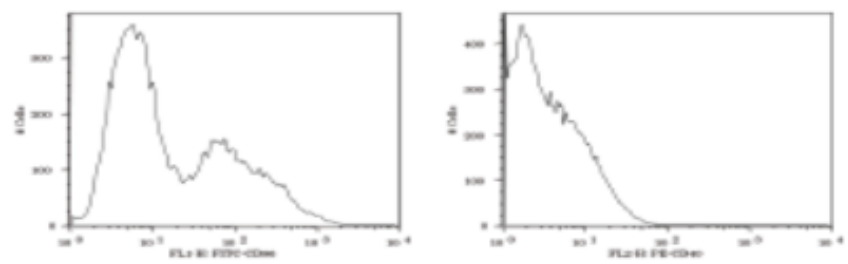

Salmon sperm

DNA
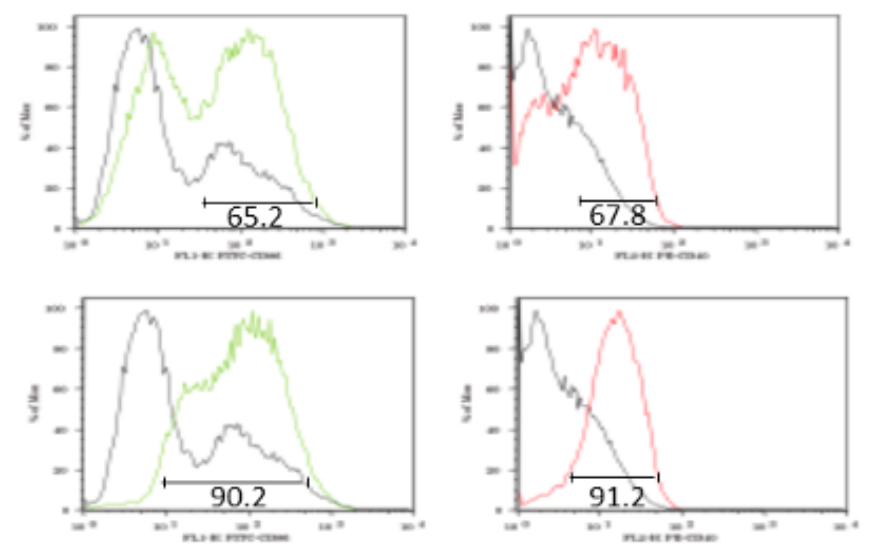

PTRE2-HBV
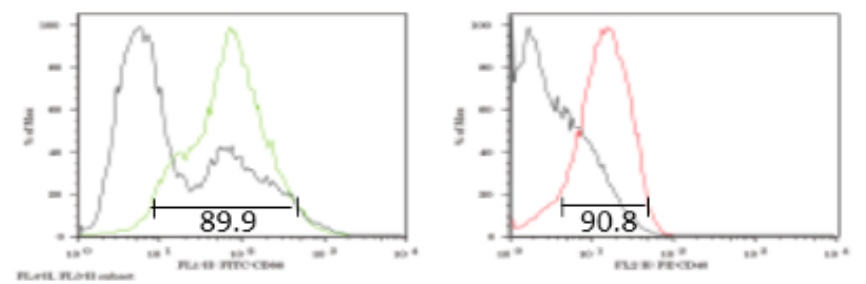

(B)
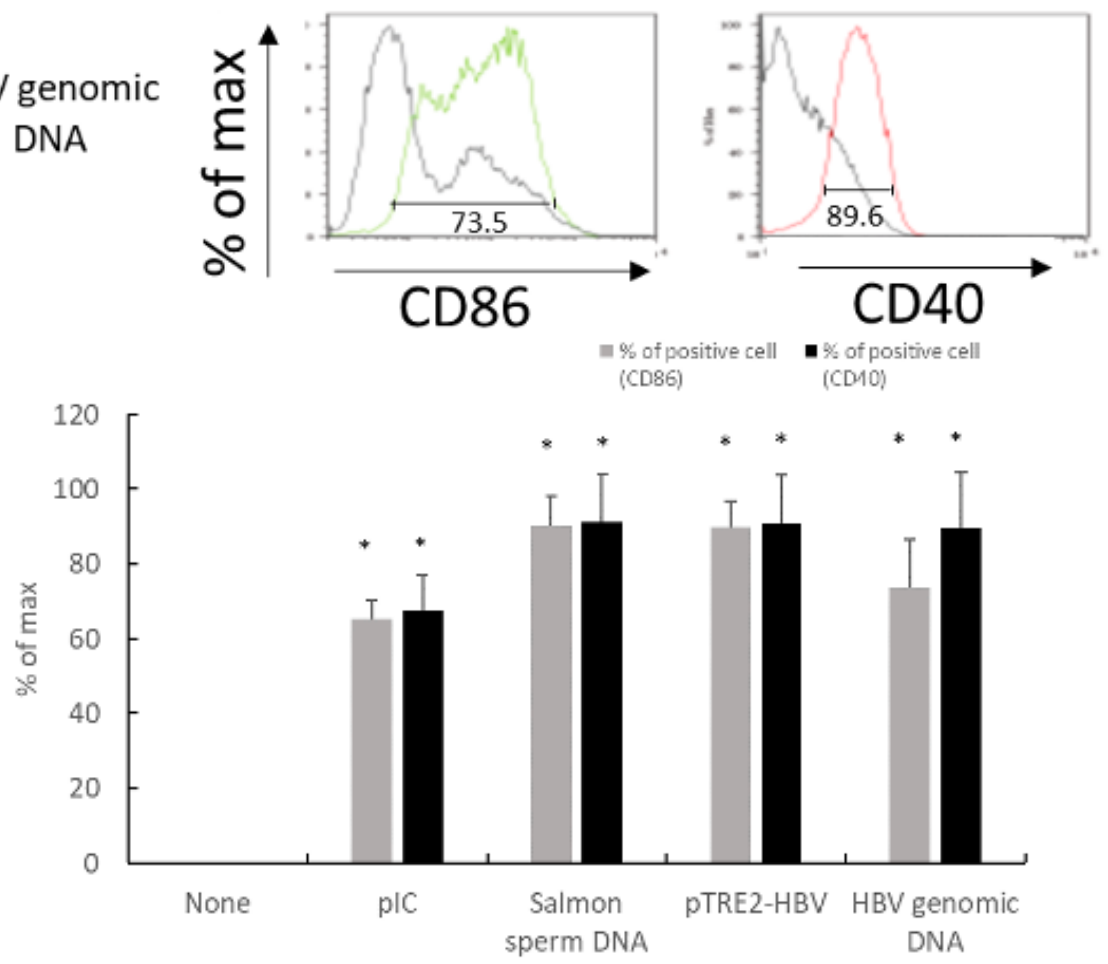

Figure 4. Transfection of BMDCs with HBV genomic DNA and a plasmid containing the HBV full genome sturdily increased the maturation of the DCs. (A) Representative flow cytometry histograms show the $\mathrm{CD} 86^{+}$(green) and $\mathrm{CD} 40^{+}$(red) BMDC population after being transfected with nucleic acid stimuli. (B) Summarized significance was determined. (Asterisks indicate a statistically significant difference between the group in comparison to the none nucleic acid stimuli group as determined by Student's t-test $* p$-value $<0.05)$. 


\section{CONCLUSION}

In this study, we found that HBV genomic DNA and SSD failed to elicit an innate immune response in HepG2 but not in RAW264.7 macrophages, indicating that hepatocytes lack a functional DNA-sensing pathway and thus are unable to respond to the presence of foreign DNA in the cytosol with a type 1 IFN response. Using BMDCs as a model, we showed that HBV genomic DNA can trigger the release of cytokines including TNF-alpha, IL-6, and IL-12p40. TLRs may be important in the uptake of extracellular viral DNA to activate BMDCs, as evidenced by the maturation and activation of the BMDCs populations upon extracellular exposure of BMDC to Poly I:C and HBV genomic DNA. Furthermore, BMDC maturation was triggered by transfection of intracellular nucleic acid stimuli, including $\mathrm{HBV}$ genomic DNA. Our findings point to DCs' crucial role in antiviral response as a possible link between the innate and adaptive immune systems during HBV pathogenesis. However, more research is needed to investigate the role of cytosol DNA sensing pathway plays in DCs during HBV infection.

\section{ACKNOWLEDGEMENTS}

The authors are thankful to Professor Kazuaki Chayama from Hiroshima University for providing the pTRE-HBV plasmid. This study is funded by the Ministry of Higher Education, Malaysia under the Fundamental Research Grant Scheme (FRGS/1/ 2017/STG03/UNIKL/02/1) and Malaysia Toray Science Foundation (MTSF).

\section{CONFLICT OF INTEREST}

The authors declare there is no conflict of interest in this study.

\section{REFERENCES}

Banchereau, J., \& Steinman, R. M. 1998. Dendritic cells and the control of immunity. Nature 392(6673): 245-252.

Barton, G. M., Kagan, J. C., \& Medzhitov, R. 2006. Intracellular localization of Toll-like receptor 9 prevents recognition of self DNA but facilitates access to viral DNA. Nature Immunology 7(1): 49-56.

Bowie, A. G., \& Unterholzner, L. 2008. Viral evasion and subversion of pattern-recognition receptor signalling. Nature Reviews Immunology 8(12): 911-922.

Buchta, C. M., \& Bishop, G. A. 2014. Toll-like receptors and B cells: functions and mechanisms. Immunologic Research 59(13): $12-22$.

Burns, S., Hardy, S. J., Buddle, J., Yong, K. L., Jones, G. E., \& Thrasher, A. J. 2004. Maturation of DC is associated with changes in motile characteristics and adherence. Cell Motility and the Cytoskeleton 57(2): 118-132.

Funami, K., Matsumoto, M., Oshiumi, H., Akazawa, T., Yamamoto, A., \& Seya, T. 2004. The cytoplasmic 'linker region'in Toll-like receptor 3 controls receptor localization and signalling. International Immunology 16(8): 1143-1154.

Ganem, D., \& Prince, A. M. 2004. Hepatitis B virus infectionnatural history and clinical consequences. New England Journal of Medicine 350(11): 1118-1129.

Hemmi, H., Takeuchi, O., Kawai, T., Kaisho, T., Sato, S., Sanjo, H., Matsumoto, M., Hoshino, K., Wagner, H., Takeda, K., \& Akira, S. 2000. A Toll-like receptor recognizes bacterial DNA. Nature 408(6813): 740-745.

Horan, K. A., Hansen, K., Jakobsen, M. R., Holm, C. K., Søby, S., Unterholzner, L., Thompson, M., West, J. A., Iversen, M. B., Rasmussen, S. B., \& Ellermann-Eriksen, S. 2013. Proteasomal degradation of herpes simplex virus capsids in macrophages releases DNA to the cytosol for recognition by DNA sensors. The Journal of Immunology 190(5): 2311 2319.

Hösel, M., Quasdorff, M., Wiegmann, K., Webb, D., Zedler, U., Broxtermann, M., Tedjokusumo, R., Esser, K., Arzberger, S., Kirschning, C. J., \& Langenkamp, A. 2009. Not interferon, but interleukin- 6 controls early gene expression in hepatitis B virus infection. Hepatology 50(6): 1773-1782.

Inaba, K., Swiggard, W. J., Steinman, R. M., Romani, N., Schuler, G., \& Brinster, C. 2009. Isolation of dendritic cells. Current Protocols in Immunology 86(1): 3-7.

Ishii, K. J., Coban, C., Kato, H., Takahashi, K., Torii, Y., Takeshita, F., Ludwig, H., Sutter, G., Suzuki, K., Hemmi, H., \& Sato, S. 2006. A Toll-like receptor-independent antiviral response induced by double-stranded B-form DNA. Nature Immunology 7(1): 40-48.

Kato, K., Ishii, R., Goto, E., Ishitani, R., Tokunaga, F., \& Nureki, O. 2013. Structural and functional analyses of DNAsensing and immune activation by human cGAS. PLOS ONE 8(10): e76983.

Kadowaki, N., Ho, S., Antonenko, S., de Waal Malefyt, R., Kastelein, R. A., Bazan, F., \& Liu, Y. J. 2001. Subsets of human dendritic cell precursors express different toll-like receptors and respond to different microbial antigens. The Journal of Experimental Medicine 194(6): 863-870.

Keating, S. E., Baran, M., \& Bowie, A. G. 2011. Cytosolic DNA sensors regulating type $\mathrm{I}$ interferon induction. Trends in Immunology 32(12): 574-581.

Kerur, N., Veettil, M. V., Sharma-Walia, N., Bottero, V., Sadagopan, S., Otageri, P., \& Chandran, B. 2011. IFI16 acts as a nuclear pathogen sensor to induce the inflammasome in response to Kaposi Sarcoma-associated herpesvirus infection. Cell Host and Microbe 9(5): 363-375. 
Kouwaki, T., Fukushima, Y., Daito, T., Sanada, T., Yamamoto, N., Mifsud, E. J., Leong, C. R., Tsukiyama-Kohara, K., Kohara, M., Matsumoto, M., \& Seya, T. 2016. Extracellular vesicles including exosomes regulate innate immune responses to hepatitis B virus infection. Frontiers in Immunology 7: 335.

Kumar, H., Kawai, T., \& Akira, S. 2009. Toll-like receptors and innate immunity. Biochemical and Biophysical Research Communications 388(4): 621-625.

Latz, E., Schoenemeyer, A., Visintin, A., Fitzgerald, K. A., Monks, B. G., Knetter, C. F., Lien, E., Nilsen, N. J., Espevik, T., \& Golenbock, D. T. 2004. TLR9 signals after translocating from the ER to CpG DNA in the lysosome. Nature Immunology 5(2): 190-198.

Leong, C. R., Oshiumi, H., Suzuki, T., Matsumoto, M., \& Seya, T. 2015. Nucleic acid sensors involved in the recognition of $\mathrm{HBV}$ in the liver-specific in vivo transfection mouse models-pattern recognition receptors and sensors for HBV. Medical Sciences 3(2): 16-24.

Leong, C. R., Funami, K., Oshiumi, H., Mengao, D., Takaki, H., Matsumoto, M., Aly, H. H., Watashi, K., Chayama, K., \& Seya, T. 2016. Interferon-stimulated gene of $20 \mathrm{kDa}$ protein (ISG20) degrades RNA of hepatitis B virus to impede the replication of $\mathrm{HBV}$ in vitro and in vivo. Oncotarget 7(42): 68179.

Leong, C. R., Oshiumi, H., Okamoto, M., Azuma, M., Takaki, H., Matsumoto, M., Chayama, K., \& Seya, T. 2015. A MAVS/TICAM-1-independent interferon-inducing pathway contributes to regulation of hepatitis B virus replication in the mouse hydrodynamic injection model. Journal of Innate Immunity 7(1): 47-58.

Lund, J., Sato, A., Akira, S., Medzhitov, R., \& Iwasaki, A. 2003. Toll-like receptor 9-mediated recognition of Herpes simplex virus-2 by plasmacytoid dendritic cells. The Journal of Experimental Medicine 198(3): 513-520.

Matsumoto, M., Funami, K., Tatematsu, M., Azuma, M., \& Seya, T. 2014. Assessment of the Toll-like receptor 3 pathway in endosomal signaling. Methods in Ensymology 535: 149-165.

Oth, T., Vanderlocht, J., Van Elssen, C. H., Bos, G. M., \& Germeraad, W. T. 2016. Pathogen-associated molecular patterns induced crosstalk between dendritic cells, $T$ helper cells, and natural killer helper cells can improve dendritic cell vaccination. Mediators of Inflammation 2016(2016): 5740373.

Phillips, S., Chokshi, S., Riva, A., Evans, A., Williams, R., \& Naoumov, N. V. 2010. CD8 ${ }^{+}$T cell control of hepatitis B virus replication: direct comparison between cytolytic and noncytolytic functions. The Journal of Immunology 184(1): $287-$ 295.

Sousa, C. R. 2006. Dendritic cells in a mature age. Nature Reviews Immunology 6(6): 476-483.

Rehermann, B., \& Nascimbeni, M. 2005. Immunology of hepatitis $\mathrm{B}$ virus and hepatitis $\mathrm{C}$ virus infection. Nature Reviews Immunology 5(3): 215-229.

Seeger, C., \& Mason, W. S. 2000. Hepatitis B virus biology. Microbiology and Molecular Biology Reviews 64(1): 51-68.

Stetson, D. B., \& Medzhitov, R. 2006. Recognition of cytosolic DNA activates an IRF3-dependent innate immune response. Immunity 24(1): 93-103.

World Health Organization, 2017. Global hepatitis report 2017. World Health Organization.

Tsuge, M., Hiraga, N., Takaishi, H., Noguchi, C., Oga, H., Imamura, M., Takahashi, S., Iwao, E., Fujimoto, Y., Ochi, H., \& Chayama, K. 2005. Infection of human hepatocyte chimeric mouse with genetically engineered hepatitis B virus. Hepatology 42(5): 1046-1054.

Thomsen, M. K., Nandakumar, R., Stadler, D., Malo, A., Valls, R. M., Wang, F., Reinert, L. S., Dagnæs-Hansen, F., Hollensen, A. K., Mikkelsen, J. G., \& Protzer, U. 2016. Lack of immunological DNA sensing in hepatocytes facilitates hepatitis B virus infection. Hepatology 64(3): 746-759.

Wieland, S. F., \& Chisari, F. V. 2005. Stealth and cunning: hepatitis B and hepatitis C viruses. Journal of Virology 79(15): 93699380.

Yin, L. K., \& Tong, K. S. 2006. Hepatitis B infection: what the primary care doctors should know. Malaysian Family Physician: The Official Journal of the Academy of Family Physicians of Malaysia 1(1): 8-10.

Zuckerman, J. N., \& Zuckerman, A. J. 2000. Current topics in hepatitis B. Journal of Infection 41(2): 130-136.

Zhang, Q. F., Shao, J. Y., Yin, W. W., Xia, Y., Chen, L., Wang, X., Hu, H. D., Hu, P., Ren, H., \& Zhang, D. Z. 2016. Altered immune profiles of natural killer cells in chronic hepatitis $B$ patients: a systematic review and meta-analysis. PLOS ONE 11(8): e0160171. 